\title{
On the Expanding Earth and Shrinking Moon
}

\author{
Golden Gadzirayi Nyambuya \\ Department of Applied Physics, National University of Science and Technology, Bulawayo, \\ Republic of Zimbabwe \\ Email: physicist.ggn@gmail.com
}

Received 18 December 2013; revised 15 January 2014; accepted 23 January 2014

Copyright (C) 2014 by author and Scientific Research Publishing Inc.

This work is licensed under the Creative Commons Attribution International License (CC BY). http://creativecommons.org/licenses/by/4.0/

(c) (i) Open Access

\begin{abstract}
Exactly 101 years ago, German scientist Alfred Lothar Wegener, sailed against the prevailing wisdom of his day when he posited that not only have the Earth's continental plates receded from each other over the course of the Earth's history, but that they are currently in a state of motion relative to one another. To explain this, Wegener set forth the hypothesis that the Earth must be expanding as a whole. Wegener's inability to provide an adequate explanation of the forces and energy source responsible for continental drift and the prevailing belief that the Earth was a rigid solid body resulted in the acrimonious dismissal of his theories. Today, that the continents are generally receding from each other is no longer a point of debate but a sacrosanct pillar of modern geology and geophysics. What is debatable is the energy source driving this phenomenon. Herein, we hold that continental drift is a result of the Earth undergoing a secular radial expansion. An expanding Earth hypothesis is currently an idea that is not accepted on a general consensus level. Be that as it may, we show herein that the law of conservation of angular momentum and energy entail that the Earth must not only expand as a consequence of the secular recession of the EarthMoon system from the Sun, but invariably, that the Moon must contract as well. As a result, the much sort for energy source driving plate tectonics can (hypothetically) be identified with the energy transfers occurring between the orbital and rotational kinetic energy of the Earth. If our calculations are to be believed-as we do; then, the Earth must be expanding radially at a paltry rate of about $+1.50 \mathrm{~mm} / \mathrm{yr}$ while the Moon is contracting radially at a relatively high rate of about $-410 \mathrm{~mm} / \mathrm{yr}$.
\end{abstract}

\section{Keywords}

Astrometry; Celestial Mechanics; Ephemerides; Planets and Satellites; Formation

\section{Introduction}

As is well known-since about the late nineteenth century [1] [2], is that, if the solid Earth did expand, one 
would in principle be able to explain the relative motion of the Earth's continental plates. The relative motion of the Earth's continental plates is technically and commonly referred to as plate tectonics ${ }^{1}$. In order that this expansion actually explain successfully the relative motion of the Earth's continental plates, it [expansion] would unambiguously have to be of the right magnitude capable of explaining the observed relative motion of the Earth's continental plates. In the this study - where the present reading is the first in a three part series, we consider the possibility that the Earth might be expanding radially as a whole solid body. This expansion is hypothesised to be driven by the observed recession of the Earth-Moon system from the Sun via spin-orbit interaction.

The laws of conservation of angular momentum and energy, when applied to the problem of the recession of the Earth-Moon system from the Sun; the pointer, strongly appears to point to the seemingly difficult-to-dismiss fact that the Earth must expand radially as a result, at a nominal rate of $\sim 1.50 \mathrm{~mm} / \mathrm{yr}$ - this is about the average growth rate of human nails [3] [4]. This same phenomenon must also occur to the Moon as well. We find that instead of expanding, the Moon must be contracting. Clearly, because we here infer the expansion of the Earth (and contraction of the Moon) from the cherished conservation laws (of angular momentum and energy), this study removes forthwith, the Expanding Earth Hypothesis (EEH) from the realm of speculation and pseudoscience, to that of plausibility. Unequivocally and unambiguously, we here put the EEH on a credible physical basis warranting further investigation.

The energy source of the expansion of the Earth and the shrinking of the Moon is not thermal in origin or emergent from any other mechanism other than the unlocking of the bound gravitational energy locked up in the orbit of the Earth-Moon system. Since the Earth-Moon system is receding from the Sun [5] [6], its energy locked up in form of orbital angular momentum must change. Because of the conservation of the total angular momentum i.e. spin and angular momentum, the change in the orbital angular momentum is transferred to the spin of the Earth-Moon system, which in-turn is transferred to the spin of the individual bodies- the Earth and the Moon. This leads to the Earth’s expansion, while the Moon contracts.

\subsection{Background}

Since about 1596, scientists have long recognised that the opposing margins of continents fit together, e.g., [7] in a manner suggesting that in the past, they formed one landmass, a hypothetical supercontinent popularly known as Rodinia ${ }^{2}$ which is thought to have formed about 1 billion years ago and to have embodied most if not all of the Earth's continents. This hypothetical supercontinent is believed to have broken up into eight continents some 600 million years ago, e.g., [8]. These eight continents later re-assembled into another supercontinent called Pangaea. Later, Pangaea broke up into Laurasia (which became North America and Eurasia of today) and Gondwana (which became the remaining continents).

Though the idea of plate tectonics had been known since about 1596, in its modern form, it was given a new birth certificate-alas, an almost stillborn birth certificate, by the perspicacity and tenacity of the now celebrated German astronomer, turned meteorologist Alfred Lothar Wegener (1880-1930). Though widely rejected by his contemporaries, Wegener [9] [10]'s idea has become the head-stone and chief-corner-stone of modern geology and geophysics. Wegener's mobilist idea of drifting continents contrasted sharply with the contemporary view of fixed and rigid continents, and a shrinking Earth due to thermodynamic cooling.

While generally not accepted, Wegener's ideas did attract a handful of respected and credible supporters such as the renowned Afrikaner geologist, Alexander Logie $d u$ Toit (1878-1948) from South Africa (whose work is summed up in the book [11]) and English geophysicist, Arthur Holmes (1890-1965) from England. At its inception, Wegener pieced together evidence from diverse fields to prop his theory. Prior to this, as early as 1910, Wegener-like others before him; realized that the continents had probably been together in the distant past, they must have drifted apart to form the arrangement that we see today. In his lifetime, Wegener wrote and published three books on the idea [12]-[14], were in each book, he sought to expound on the idea, each time making the endeavour to improve the theory in each version of the three editions. What Wegener did in his books is to present evidence of continental drift. He did not solve the problem of the source of the energy driving this drift. For this he earned sharp critism, which most certainly lead to this theory being rejected.

Despite Wegener's ideas being generally criticized on both geophysical and geological grounds, he presented a

\footnotetext{
${ }^{1}$ Tectonics is the field of geology which studies the processes which deform the Earth's crust.

${ }^{2}$ This word is derived from the Russian word rodit which means "to give birth", e.g. [8].
} 
large and significant body of very strong ponderable evidence in support of continental drift, but the mechanism driving these plates remained elusive. To this day, the energy source driving continental drift is not known and its search is one of the "holy grails" of modern plate tectonics theory. What is the energy source driving the motion of plates? For example, planets generally consist of concentric shells of matter, except for the Earth which has its unique, two-component surface, comprising of about $41 \%$ continental rock and with the reminder being quite different ocean floor basalt. To the present day, there has been no satisfactory explanation for the partial, crustal continental rock layer, except by assuming that in the distant past, the Earth was smaller and subsequently expanded [15] [16].

As already afore-stated, the principal impediment and shortcoming to this otherwise interesting idea of an expanding Earth is and has always been the lack of knowledge of a mechanism that could provide the necessary energy [17] [18] to drive this expansion without departing from the known Laws of Nature [19]. To this conundrum and quagmire, Adrian E. Scheidegger [20] stated in concisely the view:

"Thus, if expansion on the postulated scale occurred at all, a completely unknown energy source must be found."

This view, is as valid today as it was in 1982 when Scheidegger made this pronouncement. To that end, amongst others, this reading seeks to furnish the energy source driving the expansion. As we shall argue herein, this energy must be coming from the locked-up gravitational binding energy that is being unlocked in the energy transfers occurring between the orbital angular momentum and the spin angular momentum of the Earth.

In-closing the present section; from Scheidegger [20]'s comment above, it appears he (and many others) envisage a once-off expansion. As we will see herein that-like star formation is an ongoing process and not a once-off event where stars are created at a special and particular moment in the history and evolution of the Universe; the expansion of the Earth is an ongoing process that must have began at the beginning once the Earth and the Moon where formed and began their secular recession from the Sun.

\subsection{Problem Statement and Substantiation}

While it is well known that an EEH can in principle explain a number of currently inexplicable geophysical phenomenon such as plate tectonics (e.g., [21] and references therein), current scientific consensus rejects forthwith, any expansion or contraction of the Earth. However, without making an appeal to spin-orbit interaction as is done herein, but simple applying the accepted law of conservation of spin angular momentum to the well known secular increase in the length of the day as deduced by Stephenson [22]; Stephenson and Morrison [23], one can show as has been done in Ref. [24], that the Earth must be expanding at least at a rate of about $+0.60 \mathrm{~mm} / \mathrm{yr}$. Be that it may, in very recent times, the EEH is rejected on the solemn grounds of observations because on a level accuracy of about $+0.20 \mathrm{~mm} / \mathrm{yr}$, current observations using modern high-precision geodetic techniques [25] indicate that the Earth's radius has remained constant throughout its ponderable history. Before dismissing the EEH, a most logical and natural question to ask is, what is the expansion rate of the Earth that is required to explain plate tectonics?

If the expansion of the Earth required to explain plate tectonics is significantly smaller than the observed $+0.20 \mathrm{~mm} / \mathrm{yr}$ or is of the same order of magnitude as the sensitivity of the measurement, then, the current position that the Earth is not expanding requires much more sensitive measurement before sending this idea to the "Science Museum of Great but Failed Ideas". Or, if the expansion rate of the Earth is significantly less than Fukumori [6]'s measurement of $+0.20 \mathrm{~mm} / \mathrm{yr}$, then—at best, Fukumori [25]'s measurements place an upper limit to the Earth expansion and not the conclusion that they reached, that the Earth is not expanding.

As will be demonstrate herein, it appears that Fukumori [25]'s threshold of sensitivity is within the same range or order of magnitude as the signal. An application of the sacrosanct and embellished laws of conservation of momentum and energy to the Solar receding Earth-Moon system leads us to conclude that the Earth must be expanding on a scale of about $+1.50 \mathrm{~mm} / \mathrm{yr}$. In comparison, $+1.50 \mathrm{~mm} / \mathrm{yr}$ is not only about the same order of magnitude ${ }^{3}$ as Fukumori [25]'s threshold of sensitivity, the signal to sensitivity ratio is about 1:7.5—-simple, the threshold of sensitivity very low. There is thus need to improve the signal-to-sensitivity ratio to at least greater than about 1:10, thus making Fukumori [25]'s measurements inadequate to rule out an Expanding Earth Hypothesis since they are not sensitive enough to discern the implied expansion of the Earth. Placing our confidence in the law of conservation of momentum and energy, this is the position that we take.

${ }^{3}$ If the signal-sensitivity-ratio is less the 1:10, the signal is said to be of the same order of magnitude as the sensitivity. 
Therefore, the Earth may very well be expanding and the source of the energy of this expansion being the lost orbital angular momentum of the Earth-Moon system which is a result of the secular recession of this system from the Sun. If the Earth is expanding, then, evidence of this expansion should come not only in the form of relative motion of the landmasses, but also in the form of a Hubble-type motion of the landmasses. Therefore, this study is a preparatory study of a possible future study that will seek to quantity whether or not the continents are undergoing a Hubble-type flow from one another. If this can be ascertained, it would be the most revealing evidence yet that indeed, the Earth is undergoing an expansion.

Last and most important of all is that, current plate tectonics theory is incomplete in that it provides no appreciable and adequate energy source for geodynamics and it critically depends on the assumption of mantle convection. There is need to identify the energy source. It is the goal of this study to suggest or identify a plausible energy source driving the continents apart.

\subsection{Hypothesis}

That the continents are moving relative to each other is no longer a point of debate. What is debatable is the energy source driving this phenomenon. Herein, we hold that continental drift is a result of the Earth undergoing radial expansion. This expansion is driven or powered by energy transfer occurring between the rotational kinetic of the Earth's spin and the rotational orbital angular momentum kinetic energy. It is well established fact that the Moon is receding from the Earth at a rate of about $38.247 \pm 0.004 \mathrm{~mm} / \mathrm{yr}$ [26] [27], this must lead to a loss of orbital angular momentum for both the Earth and the Moon as individual bodies. In addition to this, the Russian astronomers Krasinsky and Brumberg [7] and the American astronomer Standish [12] reported that the mean Sun-Earth-Moon distance known as the Astronomical Unit (denoted $\mathrm{A} U=1.49598000 \times 10^{9} \mathrm{~m} \pm 3 \mathrm{~m}$ ) is undergoing a secular positive change of $\delta A U=150.00 \pm 3.00 \mathrm{~mm} / \mathrm{yr}$ and $\delta A U=70.00 \pm 2.00 \mathrm{~mm} / \mathrm{yr}$ respectively. To up-hold the law of conservation of angular momentum (i.e., the sum total of the orbital and rotational angular momentum), this lost orbital angular momentum can not be lost into the oblivious; it must be transferred to the spin of the respective bodies, thus leading to changes in the sizes and the spin periods of these celestial bodies since the spin depends on the size and the spin period.

\section{Corollary}

If the Earth is expanding globally, then, evidence of this expansion should come not only in the form of relative motion of the landmasses, but also in the form of a Hubble-type motion of the landmasses. That is, if one has a sphere of radius $R$ that undergoes a global radial expansion at a rate $\dot{R}$, then, any two points on the surface that are seperated by a distance $\mathcal{D}$ will undergo relative motion such that their relative rate of separation $\dot{\mathcal{D}}$ will be given by:

$$
\dot{\mathcal{D}}=\left(\frac{\dot{R}}{R}\right) \mathcal{D}
$$

The Hubble-type motion of the continental landmass assumes that the continental landmass is sitting on an expanding sphere. Therefore, a direct verification or refutation of a globally expanding Earth is to undertake a study that seeks to quantify whether or not the continents are undergoing a Hubble-type flow relative motion. A Hubble-type flow of the landmasses is but the clearest signature yet, of an expanding solid Earth. If this can be ascertained, it would be the most revealing evidence yet, that indeed, the Earth is undergoing an expansion and this expansion is causing the continents to drift apart. Hence, this work serves as a precursor or preparatory work for a future quantitative study of global plate tectonics with the aim of a verifying or refuting the hypothesis of a globally expanding Earth.

Other than a radial expansion of the Earth, it is possible that other geodynamic forces may come into play and contribute to plate tectonics such as mantle conventional currents which are thought to be the major driving force of plate motion. Lateral density variations in the mantle are believed to result in these convectional currents which if they exist, will lead to the angular displacement $(\Delta \vartheta)$ of the plates. Whatever the cause of the mantle currents, if they exist, they can be taken into account in (1) by adding an angular displacement term, that is:

$$
\dot{\mathcal{D}}=\left(\frac{\dot{R}}{R}+\frac{\dot{\vartheta}}{\vartheta}\right) \mathcal{D}
$$


If a graph of $\dot{\mathcal{D}}$ vs $\mathcal{D}$ were to be plotted, for those plates where conventional currents are not present, these will lie on a main straight line graph whose slope is $\dot{R} / R$ and passes through the origin, while for those plates where conventional currents are present-depending on the magnitude of the currents; these plates will lie off-set from the main straight line. Thus, the effect of the mantle currents on the graph $\dot{\mathcal{D}}$ vs $\mathcal{D}$ is to produce a graph with scatter of points about the main straight line graph.

Before we leave this section, it is perhaps important to make a glimpse into what to expect for the expansion rate of the Earth-we have to make a crude calculation. We know that current plate tectonic measurements indicate that the plates are moving at a rate of $\sim+10-160 \mathrm{~mm} / \mathrm{yr}$. According to our proposed Hubble type expansion theory as laid down above, if we assumed that the angular component is negligible, i.e. $\dot{\vartheta}=0$, and given that the average distance between continental margins should be about $1 / 12^{\text {th }}$ of the circumference of the Earth (i.e., $\mathcal{D} \sim 5-10,000 \mathrm{~km}$, which corresponds an angular displacement $\Delta \vartheta \sim 30^{\circ}$ ), from this crude information and the Hubble type expansion model of the Earth, the expected expansion rate of the Earth must be of the order of $+1-100 \mathrm{~mm} / \mathrm{yr}$. So, as we workout the implied expansion rate from the conservation of energy and angular momentum, we shall keep at the back of our mind the afore-calculated expansion rate-this will act more as a guide.

\subsection{Aim}

This study explores the viable possibility of a secular expansion of the Earth. This secular expansion is hypothesised to be a direct consequence of the observed secular recession of the Earth from the Sun. The supreme and paramount aim of the study is to suggest or posit that the observed continental drift may very well be a result of an expanding Earth.

\subsection{Objectives}

The objectives of this study (as enshrined in the three part series of readings) are as follows:

1. Judiciously apply the law of conservation of angular momentum to the Earth-Moon system - posteriori justified; the sole aim of which is to unearth a plausible astrophysical link to global geo-plate tectonics.

2. Calculate the implied radial expansion and or contraction of the of the Earth and the Moon.

3. Show that the rotational kinetic of the Earth-Moon system is a viable, potent and plausible source energy capable of driving global plate tectonics.

4. By way of a literature study, set the stage for further work on the possibility of an astrophysical link to global geo-plate tectonics.

5. Present a new plausible theory on the way the Earth-Moon system might have formed. A backward extrapolation of the evolution of the Earth-Moon system for an expanding Earth, contracting Moon and a receding Earth-Moon leads to a very interesting paradigm that might explain many puzzles about the nature of the Earth-Moon system.

\subsection{Justification}

Since the idea of plate tectonics was given birth to, the source driving this activity has eluded those that have made the endeavour to decipher the source. Finding this source is not only of great importance to geology and geophysics, but to science in general because it would lead to a better understanding of the forces operating in our terrestrial habitat. Thus, the paramount nature and justification of the present endeavour cannot be under or overstated.

\subsection{Approach to Problem}

Our present approach to the problem of finding a plausible energy source that is driving the drifting of continental plates is purely a theoretical one; where-after, it is anticipated that data will be gathered (from existing geophysical stations and from Global Positioning System satellites) in the near future to consolidate the ideas propagated herein. We demonstrate that from the embellished, sacrosanct and cherished law of conservation of angular momentum and energy, that, the Earth is expected to expand radially if the Earth-Moon system is receding from the Sun as determined from the observations of Pitjeva; Standish; Krasinsky and Brumberg [5] [6] [28]. 


\subsection{Motivation}

Only until recently, it has become clear that our understanding of the gravitational phenomenon is in serious dearth. The emerging picture was now that where our knowledge of the gravitational phenomenon appeared to be taking a good shape. Alas, the opposite is actually the case. This position has been brought about because of the improvement in our advancement in technology. There has been a recent upsurge of gravitational "anomalies" and this is a direct result of the higher resolution measurements brought by the aforesaid technological advancements. Gravitational anomalies have puzzled the scientific community for quite sometime now.

First, was the discovery of the so-called darkmatter by the Swiss astronomer Fritz [29], followed by the Pioneer anomaly in the late 1980's by the United States of America's National Aeronautic Space Administration (NASA) scientists Anderson et al. [30] [31], then come the Earth-flyby anomalies in the early 1990's again by NASA scientists [32] and more recently, the secular increase in the mean Earth-Sun and mean Earth-Moon distance by independent groups of American and Russian [5] [6] [26] [28]. What really is going on with gravitation? What is the matter? Do we really understand gravitation? Why suddenly an upsurge of these gravitational anomalies? For a conscience review of Solar gravitational anomalies (see e.g. [33]).

The widely accepted and dominate gravitational model in contemporary physics is Einstein [34]'s General Theory of Relativity (GTR). Against the desideratum, Einstein's GTR is unable to deliver non-ad hoc and nonimpromptu solutions to these problems. That the need for new fresh ideas on this front is imminent, is something that few researchers on these frontiers doubt not, but feel strongly that, this is the way forward. Our motivation is thus the desire to understand the gravitational phenomenon by seeking improved models of gravitation that are able to explain most if not all of these gravitational anomalies from a unified standpoint, that is, explain these using one model and not models designed only to address a particular anomaly.

\subsection{Synopsis}

The present reading is organised as follows. In the subsequent section, we lay down the proposed theory that we put forward as a plausible explanation of global plate motion. It is not a new theory in the traditional sense of bringing new exotic concepts, but merely a direct application of the law of conservation of angular momentum on the Earth-Moon system. In $\S(3)$, we make preparatory calculations that are necessary to quantity the 'new' ideas. In $\S(4)$, we derive a quantifiable formula giving the expansion rate of the Earth. In $\S(5)$, we apply the 'new' ideas to physically measured data. In our application, we check if the 'newly' founded ideas yield reasonable physical quantities that are in reasonable correspondence with experience. Lastly, in $\S(6)$, we give a general discussion, the conclusions drawn thereof and the recommendations that we feel can be taken up by future studies.

\section{Theory}

Both Newtonian and Einsteinian gravitation assume that the orbital angular momentum of a planet orbiting the Sun must be a conserved quantity, thus the emergence of the recession of the Earth-Moon system from the Sun and as-well the recession of the Moon from the Earth has come as nothing short of a Newtonian and Einsteinian surprise. Recessional motion of the Earth-Moon system and the Moon from the Earth have implications on the angular momentum. Since the magnitude of the orbital angular momentum $\mathcal{J}$ depends on the mass $\mathcal{M}$, orbital radius $\mathcal{R}$, and the period $\mathcal{T}$, that is $\mathcal{J}=2 \pi \mathcal{M} \mathcal{R}^{2} / \mathcal{T}$, if $\dot{\mathcal{J}}=0$, then, either the mass or the orbital period must change so as to compensate for the change in orbital radius. In the case of Solar planet, their masses can be considered to be constant, thus this leaves the period as the only quantity to vary in-order to compensate for the change in orbital radius. Prima facie, it would then appear as though the sacrosanct law of conservation of angular momentum is here being violated. However, a closer inspection will reveal that this is not the case. In general, what must be conserved is not the orbital angular momentum but the total angular momentum. The total angular momentum $(\mathcal{L})$ includes two kinds of angular momentums, i.e. the orbital angular momentum $(\mathcal{J})$ and the spin angular momentum $(\mathcal{S})$, i.e. $\quad \mathcal{L}=\mathcal{J}+\mathcal{S}$ is what must be conserved, i.e. $\dot{\mathcal{L}}=0$. This means that $\dot{\mathcal{J}}=-\dot{\mathcal{S}}$; this can be rewritten in a more convenient way as:

$$
\frac{\dot{J}}{J}=-\left(\frac{S}{J}\right) \frac{\dot{\boldsymbol{S}}}{S}-\left(\frac{\dot{\mathcal{M}}}{\mathcal{M}}\right) \frac{\boldsymbol{J}+\boldsymbol{S}}{J},
$$


where $\boldsymbol{J}$ and $\boldsymbol{S}$ are the specific orbital angular momentum and the spin-by specific, we mean per unit mass.

In the reading [35] where an alternative model of gravitation coined the Azimuthally Symmetric Theory of Gravitation (hereafter ASTG-model) has been posited; after the realisation that the ASTG-model required that the orbital angular momentum be not a conserved quantity, the above idea that the total angular momentum is what must be conserved was proposed as a necessary, straight forward and logical manner to preserve the law of conservation of angular momentum. This proposal does not in any way violate any of the known Laws of Physics but is wholesomely and completely within the framework and realm of the known Laws of Physics. The added and interesting outcome of this is that, it brings into effect the possibility of spin-orbit interaction. This idea is the central theme of the present reading.

The next central idea is the conservation of energy. An object orbiting some central massive body is going to have some rotation kinetic energy $K_{\text {orb }}$ and as well some gravitational potential energy $U_{\text {orb }}$. The total energy associated with its orbit about this central massive body $E_{\text {orb }}=K_{\text {orb }}+U_{\text {orb }}$, is conserved, i.e. $\dot{E}_{\text {orb }}=0$. From this is follows that:

$$
K_{\text {orb }}\left(\frac{\dot{K}_{\text {orb }}}{K_{\text {orb }}}\right)+U_{\text {orb }}\left(\frac{\dot{U}_{\text {orb }}}{U_{\text {orb }}}\right)=0 .
$$

Actually, the assumption of the conservation of the total energy associated with the orbit of test body is central not only to Newtonian gravitational but Einsteinian gravitation as-well, so this idea is sound and valid.

The next conservation is that of the energy associated with the spin of the orbiting and spinning test body. From what has been stated above, the rotational orbital kinetic energy $K_{\text {orb }}$ interacts in a conserved manner with the gravitational potential energy $U_{\text {orb }}$ of the test body. In the same manner, the spin must interact with the total stored gravitational energy $U_{\mathrm{g}}$ i.e., for an object of mass and radius $\mathcal{M}_{\mathrm{obj}}$ and $\mathcal{R}_{\mathrm{obj}}$ respectively, the total stored gravitational energy $U_{\mathrm{g}}=-G \mathcal{M}_{\mathrm{obj}}^{2} / \mathcal{R}_{\mathrm{obj}}$. The total energy $E_{\text {spin }}=K_{\mathrm{spin}}+U_{\mathrm{g}}$, must conserved, i.e. $\dot{E}_{\text {spin }}=0$. From this is follows that:

$$
K_{\text {spin }}\left(\frac{\dot{K}_{\text {spin }}}{K_{\text {spin }}}\right)+U_{\mathrm{g}}\left(\frac{\dot{U}_{\mathrm{g}}}{U_{\mathrm{g}}}\right)=0 .
$$

Thus the present theory is summed up in the three conservation Equations (3), (4) and (5). Undoubtedly, the foundation of our theory is not without a firm and valid basis.

\section{Preliminary Computations}

We are now going to apply the ideas of the presiding section to the Sun-Earth-Moon system. Our first port of call is to establish a single value for the recession of the Earth-Moon system from the Sun. The mean distance from the Sun of the Earth-Moon system is referred to as the Astronomical Unit and denoted by the symbol $A U$. Let us represent the secular change in the Astronomical Unit by $\delta A U$. As stated earlier, at present, there are two values for this quantity, that is, the Russian astronomers Krasinsky and Brumberg [5] find $\delta A U=+150.00 \pm 3.00$ $\mathrm{mm} / \mathrm{yr}$, while the American astronomer Standish [6] finds $\delta A U=+70.00 \pm 2.00 \mathrm{~mm} / \mathrm{yr}$. From these two values we need the best estimate. For this, we need to appeal to statistical methods to find a best estimate.

Assuming that these two measurements are governed by Gaussian statistics and that the errors in the measurements random and independent, then, the best estimate of these two measurements can be obtained by taking the weighted mean of the two values. For example if $\left(x_{i}+\delta x_{i}: i=1,2, \cdots, n\right)$ is set of $n$ measurements of a constant quantity $x$, where $x_{i}$ is the best value of for the $n^{\text {th }}$ measurement and $\delta x_{i}$ is its accompanying error margin, then, the best estimate of $x_{\text {best }}$ from this set is $x_{\text {best }}=\sum w_{i} x_{i} / \sum w_{i}$ where $w_{i}$ are the weights such that $w_{i}=1 /\left(\delta x_{i}\right)^{2}$ and the best estimate in the error margin $\delta x_{\text {best }}$ is $\delta x_{\text {best }}=\left(\sum w_{i}\right)^{-1 / 2}$ (see e.g. [36] p. 150). Applying this prescription to the two measurements of Standish; Krasinsky and Brumberg [5] [6], we obtain:

$$
\delta A U=+95.00 \pm 2.00 \mathrm{~mm} / \mathrm{yr} .
$$

We shall from heron adopt this value (6) as representative of the change in the mean distance between the Sun and Earth-Moon system.

The maximum distance of the Earth from the Sun $\mathcal{R}_{\text {orb }}^{\max }=1.52098232 \times 10^{11} \mathrm{~m}$ and minimum distance is $\mathcal{R}_{\text {orb }}^{\min }=1.47098290 \times 10^{11} \mathrm{~m}$ [37]. In our calculation, we need one single value for the mean distance between 
the Sun and the Earth-Moon system. From $\mathcal{R}_{\text {orb }}^{\min }$ and $\mathcal{R}_{\text {orb }}^{\max }$, the best estimate would the average of these two values, that is, $\mathcal{R}_{\text {orb }}^{\text {best }}=\left(\mathcal{R}_{\text {orb }}^{\max }+\mathcal{R}_{\text {orb }}^{\max }\right) / 2$ and the best estimate in the error margin to this value is $\delta \mathcal{R}_{\text {orb }}^{\text {best }}=\left(\mathcal{R}_{\text {orb }}^{\max }-\mathcal{R}_{\text {orb }}^{\text {max }}\right) / 2$, so that the best value for the mean distance between the Sun and the Earth-Moon system is:

$$
\mathcal{R}_{\text {orb }}=(1.50 \pm 0.03) \times 10^{11} \mathrm{~m} .
$$

Now, lets compute the spin angular momentum of the Earth-Moon system $S_{\text {em }}=2 \pi\left(\mathcal{M}_{\mathrm{e}}+\mathcal{M}_{\mathrm{m}}\right) \mathcal{R}_{\mathrm{em}}^{2} / \mathcal{T}_{\text {em }}$. The mass of the Earth $\mathcal{M}_{\mathrm{e}}$ and the Moon $\mathcal{M}_{\mathrm{m}}$ are estimated to $5.9736 \times 10^{24} \mathrm{~kg}$ and $7.3477 \times 10^{22} \mathrm{~kg}$ respectively. The period $\mathcal{T}_{\text {em }}$ which is the mean period of the Moon in its orbit around the Earth is estimated to be $27.321582 / 365.25 y r$. In order to calculate $\mathcal{S}_{\text {em }}$, we are left with the value of $\mathcal{R}_{\mathrm{em}}$. At perigee, we have $\mathcal{R}_{\mathrm{em}}^{\min }=3.62570 \times 10^{8} \mathrm{~m}$ and at apogee, we have $\mathcal{R}_{\mathrm{em}}^{\max }=4.05410 \times 10^{8} \mathrm{~m}$. Using the same procedure that we used to estimate the best value for the mean distance between the Sun and the Earth-Moon system, we have for the mean distance between Earth and Moon $\mathcal{R}_{\mathrm{em}}=(3.80 \pm 0.20) \times 10^{8} \mathrm{~m}$.

\section{Derivation}

We here derive the expansion rate formula for the Earth from the two concepts to be used the conservation of angular momentum and energy of the Earth-Moon system. The total angular momentum of the Earth-Moon system comprises six components, that is: (1) the orbital angular momentum of the Earth-Moon system $\mathcal{J}_{\text {orb }}$ about Solar center of mass, (2) the reduced mass term of the system $\mathcal{J}_{\text {rm }}$, (3) the orbital angular momentum of the Earth about the common center of mass $\mathcal{J}_{\mathrm{e}}$, (4) the orbital angular momentum of the Moon about the common center of mass $\mathcal{J}_{\mathrm{m}}$, (5) the spin angular momentum of the Earth about its center of mass $\mathcal{S}_{\mathrm{e}}$ and lastly, (6) the spin angular momentum of the Moon about its center of mass $\mathcal{S}_{\mathrm{e}}$. So, the total angular momentum $\mathcal{L}$ is given by:

$$
\mathcal{L}=\mathcal{J}_{\text {orb }}+\mathcal{J}_{\text {rm }}+\mathcal{J}_{\mathrm{e}}+\mathcal{J}_{\mathrm{m}}+\mathcal{S}_{\mathrm{e}}+\mathcal{S}_{\mathrm{m}}=i \mathcal{L}_{x}+j \mathcal{L}_{y}
$$

In the about, the components $\mathcal{L}_{x}$ and $\mathcal{L}_{y}$ are the $x$ and $y$ components of $\mathcal{L}$ and from Figure 1 , one can deduce that these components are such that:

$$
\begin{aligned}
& \mathcal{L}_{x}=\mathcal{S}_{\mathrm{m}} \sin \vartheta_{\mathrm{m}}-\mathcal{S}_{\mathrm{e}} \sin \vartheta_{\mathrm{e}}+J_{\text {orb }} \sin \vartheta_{\text {orb }}, \\
& \mathcal{L}_{y}=\mathcal{J}_{\mathrm{rm}}+\mathcal{J}_{\mathrm{e}}+\mathcal{J}_{\mathrm{m}}+\mathcal{S}_{\mathrm{m}} \cos \vartheta_{\mathrm{m}}-\mathcal{S}_{\mathrm{e}} \cos \vartheta_{\mathrm{e}}+J_{\text {orb }} \cos \vartheta_{\text {orb }} .
\end{aligned}
$$

For our purpose here, we shall make a simplifying assumption, namely that the axial tilt of both the Earth and the Moon has remained constant, that is, it does not change, this means we shall take $\dot{\vartheta}_{\mathrm{e}}=\dot{\vartheta}_{\mathrm{m}}=\dot{\vartheta}_{\text {orb }}=0$. It is very much possible and highly likely that the axial tilt angle of the Earth and Moon have changed over the course of their history and that currently, much in the same manner as other Solar secular changes, the axial tilt

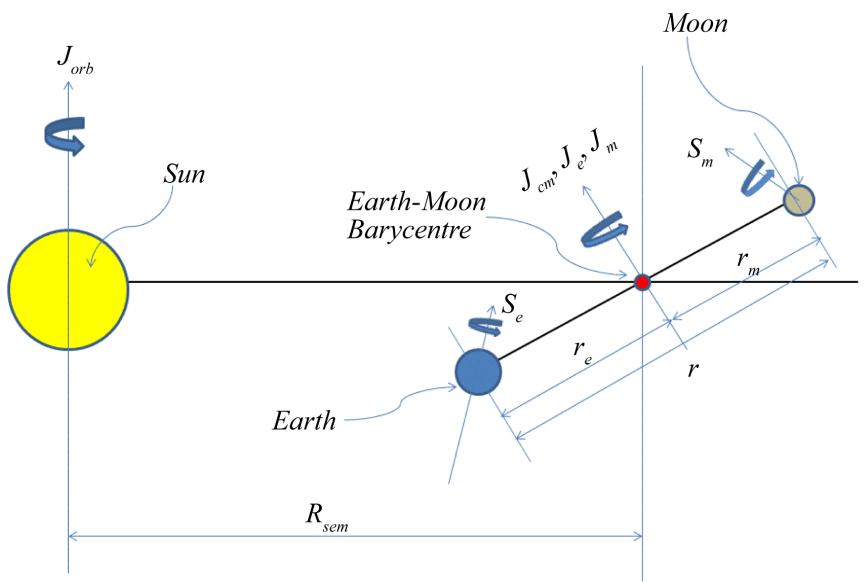

Figure 1. A schematic diagram of the Sun-Earth-Moon System showing the orientations and directions of the spins and orbital angular momentum of these celestial bodies. 
angle of these celestial bodies is changing. In the present expedition, we find no reason to invoke this possibility, thus, we simple let it to the rest, in which event, the resulting calculations are much easier to handle.

Now, let us define the quantity $L$, which is such that:

$$
L_{\mathrm{e}}=\cos \vartheta_{\mathrm{m}} \mathcal{L}_{x}-\sin \vartheta_{\mathrm{m}} \mathcal{L}_{y} .
$$

Written in full, $L$ is as given in (A.20). From the above assumptions that $\dot{\vartheta}_{\mathrm{e}}=\dot{\vartheta}_{\mathrm{m}}=\dot{\vartheta}_{\text {orb }}=0$, and as-well from the law of conservation of angular momentum $\left(\dot{\mathcal{L}}=0: \Rightarrow \dot{\mathcal{L}}_{x}=\dot{\mathcal{L}}_{x}=0\right)$, it follows that $\dot{L}=0$. Using these facts in conjunction with the law of conservation of energy, the sought for formula is derived overleaf from (A.20) right to (A.25). In the next section, we shall apply this result to compute the predicted expansion rate of the Earth.

\section{Results and Analysis}

We give here the results emerging from the derived formulae (A.25) and (A.26) for the Earth and the Moon systems in section (5.1) and (5.2) respectively. In section (5.3), we give an analysis of the results.

\subsection{Earth System}

Now, from (A.25), the expansion terms can be split into two, that is, Solar and Lunar contribution terms, that is:

$$
\frac{\delta \mathcal{R}_{\mathrm{e}}}{\mathcal{R}_{\mathrm{e}}}=\left(\frac{\delta \mathcal{R}_{\mathrm{e}}}{\mathcal{R}_{\mathrm{e}}}\right)_{\odot}+\left(\frac{\delta \mathcal{R}_{\mathrm{e}}}{\mathcal{R}_{\mathrm{e}}}\right)_{L}
$$

By inserting the relevant values, one finds that for the Solar contribution, we will have:

$$
\left(\frac{\delta \mathcal{R}_{\mathrm{e}}}{\mathcal{R}_{\mathrm{e}}}\right)_{\odot}=+(2.40 \pm 0.20) \times 10^{-10} \mathrm{yr}^{-1},
$$

and for the Lunar contribution, we will have:

$$
\left(\frac{\delta \mathcal{R}_{\mathrm{e}}}{\mathcal{R}_{\mathrm{e}}}\right)_{L}=+(5.10 \pm 0.50) \times 10^{-13} \mathrm{yr}^{-1} .
$$

Clearly, the Lunar contribution is far weaker than the Solar contribution, it is so weak so much that, we can, for our purposes here, neglect it altogether. From this, we can safely conclude that, whatever force is driving the recession of the Earth-Moon system from the Sun, it is this same phenomenon that should lead to the Earth to expand. Therefore, the recession of the Earth-Moon system from the Sun leads to the expansion of the Earth which in-turn leads to plate tectonics. From (13), the corresponding rate of expansion of the Earth is:

$$
\delta \mathcal{R}_{\mathrm{e}}=+1.50 \pm 0.10 \mathrm{~mm} / \mathrm{yr} \text {. }
$$

\subsection{Moon System}

At its conception, this reading never meant to touch seriously on matters to do with the Moon. Actually, after we had calculated and demonstrated that the Earth might be expanding, we felt that was enough, the reading must be sent off for publication. On second thoughts, we felt that the reviewers might ask, "Since this is within the realm of your calculation, what does your calculation give for the Moon?”. It is only then, that we felt, we must address this matter before we are compelled to do so. We had envisaged a simple reading where we report that the Earth-Moon drift from the Sun, most certainly is the driver to geo-plate tectonics. The same calculation as that for the expansion rate of the Earth, was conducted for the Moon when upon we arrived as (A.26).

Substituting the relevant figures into this formula, one notices that as in the case of the Earth, the Lunar recession's contribution is minute compared to that Solar recession term, that is:

$$
\left(\frac{\delta \mathcal{R}_{\mathrm{m}}}{\mathcal{R}_{\mathrm{m}}}\right)_{\odot}=-(2.35 \pm 0.20) \times 10^{-7} \mathrm{yr}^{-1},
$$

and: 


$$
\left(\frac{\delta \mathcal{R}_{\mathrm{m}}}{\mathcal{R}_{\mathrm{m}}}\right)_{L}=+(1.38 \pm 0.20) \times 10^{-10} \mathrm{yr}^{-1}
$$

The Lunar recessional term leads to an expansion of $+0.20 \mathrm{~mm} / \mathrm{yr}$ while the Solar recessional term leads to a contraction of $\sim-410 \mathrm{~mm} / \mathrm{yr}$. Obviously, the expansion is dwarfed by the contraction so that overall, the Moon must be in a state of contracting. Precisely, one find that:

$$
\delta R_{\mathrm{m}}=-410.00 \pm 0.10 \mathrm{~mm} / \mathrm{yr} \text {. }
$$

At the time we performed this calculation, our ideas of the geology of the Moon where the pre-2010 ideas; we thought the Moon must be a geological dead piece of rock. Our first reaction to the idea that the Moon might be contracting was that of scepticism. The Moon can not be contracting, especially at such a significant rate of $\sim-410.00 \pm 0.10 \mathrm{~mm} / \mathrm{yr}$.

As any researcher would do nowadays, we "hit" google. Much to our surprise, elation and delight, we found out that only recently have Watters et al. [38] found the first evidence that the Moon is undergoing global contraction. Watters et al. [38] reported that this evidence has been brought forth by NASA's Lunar Reconnaissance Orbiter Camera (LROC), launched in 2009 aboard the Lunar Reconnaissance Orbiter-which is the first spacecraft to be launched by NASA as part of it's "Return to the Moon" initiative.

The LROC photographed Lunar scars known as lobate scarps. Lobate scarps occur when the surface of the geological body experiences a compressional force, causing one part of the upper surface to fold and fracture above the other part. In the absence of significant tectonics on the Moon, Watters et al. [38] believe this is due to cooling of the Lunar core. If our ideas are correct or prove to be correct in the future, then, this contraction is not due to cooling as Watters et al. [38] believe, but gravitational contraction caused by the transfer of orbital angular momentum into spin angular momentum and this been a result of the observed secular recession of the Earth-Moon system. As the core of the Moon cooled it also shrunk, applying surface stress to the brittle Lunar crust and causing it to rupture and split.

Lobate scarps have been observed on the surface of the Moon before i.e., from images taken by the panoramic cameras aboard the Apollo 15, 16 and 17 missions Watters et al.; Binder and Gunga [38] [39]. These earlier missions were confined to the equatorial region of the Moon's surface thus giving not a picture of this being a global phenomenon [39]. Using the LROC, Watters et al. [38] managed to acquire comprehensive images of the Lunar surface at higher latitudes. The lobate scarps seem to have formed relatively recent-about a billion years or so Watters et al. [38] and the most recent analysis by Banks et al. [40] of these lobate scarps further points to Lunar global contraction.

Other than lobate scarps, the Lunar surface has recently been shown to contain a significant number of grabens [41]. Grabens are tectonic features that form under extension stresses or tension of the landmass. Structurally, they are comprised of two normally intersecting faults, with a down-dropped block between them. Most grabens are found within the Lunar maria near the edges of large impact basins. Despite the presence of these grabens, Watters et al. [41] believe the Moon should still be undergoing global contraction. Of this (i.e., Lunar contraction), the lead author from the United State of America's Center for Earth and Planetary Studies, Thomas Watters had this to say:

"We think the Moon is in a general state of global contraction due to cooling of a still hot interior. The graben tell us that forces acting to shrink the Moon were overcome in places by forces acting to pull it apart. This means the contractional forces shrinking the Moon cannot be large, or the small graben might never form. ${ }^{4}$

In conclusion on the matter of Lunar contraction, i.e., the Lunar contraction rate as calculated herein, we have this to say. While we are very skeptical of a Lunar contraction rate as high as $\sim 410 \mathrm{~mm} / \mathrm{yr}$, if we have to learn from history, we have to place aside our skepticism and accept the result pending verification or refutation from observations. This calculation is inferred from the law of conservation of angular momentum and energy, we have no choice but to place, not our faith in the calculation, but our confidence in the calculation, confidence that, it is highly likely that this result will in the near future be verified. Our skepticism or any that can be brought forth is nothing but skepticism whose strong foundations is based on prejudice of a scientific nature.

Is it not scientific prejudice that denied Albert Einstein (1879-1955) the monumental and once in a lifetime opportunity to predict that the Universe might be expanding? Is it not scientific prejudice that stalled Wegener's

\footnotetext{
${ }^{4}$ Quote from http://newsdesk.si.edu/releases/new-images-show-recent-geologic-activity-moon.

Accessed on this day 4/12/2012 15h04 GMT + 2 .
} 
hypothesis of continental drift? Is it not scientific prejudice that might have lead to the rejection of the celebrated French Prince, Louis Victor Peirre Raymond de Brogile (1872-1946)'s wave-particle duality leading to a great stagnation in the developed of quantum mechanics? While not endless, the list is long. Sometimes, we must accept the bare facts before us, and if Nature is to embarrass us, we must be humble enough to accept this as an honour rather than a dishonour. We would rather be "embarrassed" by Nature than by anything else. Nature is the greatest teacher of all, we must learn from the best.

\subsection{Energy Source for Platetectonics}

The energy to drive plate tectonics must come from the energy locked-up in the spin of the Earth. This energy is unlocked when the Earth-Moon system recedes from the Sun. The total energy locked up in the spin is $K_{\text {spin }}^{\mathrm{e}}=\mathcal{S}_{\mathrm{e}}^{2} / 2 \mathcal{M}_{\mathrm{e}} \sim+5.20 \times 10^{29} \mathrm{~J}$. When the Earth expands, energy is drawn at a rate $\delta K_{\text {spin }}^{\mathrm{e}}=\mathcal{S}_{\mathrm{e}} \delta \mathcal{S}_{\mathrm{e}} / \mathcal{M}_{\mathrm{e}}$, that is:

$$
\delta K_{\text {spin }}^{\mathrm{e}}=\frac{8 \pi^{2} \mathcal{M}_{\mathrm{e}} \mathcal{R}_{\mathrm{e}}^{4}}{\mathcal{T}_{\mathrm{e}}^{2}} \frac{\delta \mathcal{R}_{\mathrm{e}}}{\mathcal{R}_{\mathrm{e}}}=+2.45 \times 10^{34} \mathrm{Jyr}^{-1} .
$$

This is at a rate of about $+2.40\left|\mathcal{L}_{\odot}\right|$, that is, the power driving plate tectonics is about two and half times the power of the Sun.

In the case of the Moon which is must be contracting at a rate of $\sim-410 \mathrm{~mm} / \mathrm{yr}$, it must be giving off energy at a rate $\sim-7.10 \times 10^{22} \mathrm{Js}^{-1}=0.0002 \mathcal{L}_{\odot}$.

All this energy that drives the expansion of the Earth and the contraction of the Moon is drawn from the lost orbital kinetic energy of the Earth-Moon system $K_{\text {orb }}=\mathcal{J}_{\text {orb }}^{2} / 2\left(\mathcal{M}_{\mathrm{e}}+\mathcal{M}_{\mathrm{m}}\right)$. This orbital kinetic energy of the Earth-Moon system is given of at a rate $\delta K_{\text {orb }}=\mathcal{J}_{\text {orb }} \delta \mathcal{J}_{\text {orb }} /\left(\mathcal{M}_{\mathrm{e}}+\mathcal{M}_{\mathrm{m}}\right)=+4.90 \times 10^{36} \mathrm{Js}^{-1}=+1.50 \times 10^{10}\left|\mathcal{L}_{\odot}\right|$, this is of the order of the luminosity of a typical galaxy! Clearly, there is more than enough energy to drive the Earth's plates apart. This recession of the Earth-Moon system certainly unlocks a significant amount of energy from the orbital kinetic energy. Besides there being enough energy from the lost orbital kinetic energy of the Earth-Moon system via the Earth-Moon Solar drift, this energy (from the Earth-Moon Solar drift) far outweighs the internal energies of the two systems so much that one can safely ignore these energies without making a critical omission e.g. these energies are (1) the energy locked up in the Earth-Moon tides and, (2) the mass loss via energy from radiative matter in the Earth and as-well the Moon.

For example, the Earth's internal heat comes from a combination of factors, the dominant of which are the residual heat from planetary accretion which contributes about $20 \%$ of the Earth's heat, and the rest produced through radioactive decay which contributes about $80 \%$ (see e.g., [42] pp. 136-137). For the radioactive heat production, the major heat-producing isotopes Potassium-40 $\left(\mathrm{K}^{40}\right)$, Uranium-238 ( $\left.\mathrm{U}^{238}\right)$, Uranium- 235 $\left(\mathrm{U}^{235}\right)$, and Thorium- $232\left(\mathrm{Th}^{232}\right)$. On average, heat loss from the Earth is $\sim 0.0087 \mathrm{Wm}^{-2}$ and for the entire Earth, the global heat loss is $4.42 \times 10^{13} \mathrm{~W}$ (see e.g. [43]). Measured in units of Solar luminosity, the energy loss $4.42 \times 10^{13} \mathrm{~W}$ is $1.36 \times 10^{-13} \mathcal{L}_{\odot}$. In compassion to the energy unleashed from the Earth-Moon recession $\left(+1.50 \times 10^{10}\left|\mathcal{L}_{\odot}\right|\right)$, and from that which goes into the expansion of the Earth $\left(+2.40\left|\mathcal{L}_{\odot}\right|\right)$ as a result of spin-orbit interaction the energy from the heat loss $\left(1.36 \times 10^{-13} \mathcal{L}_{\odot}\right)$ is insignificantly small.

It has been urged in [24] that mass loss of the Earth must lead to the expansion of the Earth if the spin angular momentum is to be conserved separately. Heat loss must according to Einstein's mass energy equivalence lead to a mass loss of the Earth. If one applies the formula in Equation (1) of [24] to the mass loss rate from the Earth's heat loss, they will conclude that the expected radial expansion of the Earth must be $\sim+8.00 \times 10^{-12}$ $\mathrm{mm} / \mathrm{yr}$. On any scale, this is insignificantly small. Clearly, the internal energy changes occurring inside the Earth are of no consequence and can safely be ignored when one is considering energies associated with the possible expansion of the Earth. The same goes for the energy associated with Lunar-Earth tides, it is insignificantly small so much that when one is considering energies associated with the possible expansion of the Earth, they can safely ignore this without committing a significant error in their analysis.

\section{Discussion, Conclusion and Recommendations}

Below we present a general discussion, the conclusion drawn thereof, and the recommendations we make for future studies and endeavours toward investigations into the possibility that the Earth might actually be under- 
going a secular expansion as inferred herein.

\subsection{General Discussion}

Judiciously and with great equanimity, allow us to say this at the outset of the present section, namely that, while the ideas propagated herein spring-forth from non-exotic nor exogenous ideas but directly from the provinces of the well accepted Physical Laws, if proven correct-as we strongly believe they will; it goes without saying that their implications are nothing short of asymptotically pivotal insofar as our understanding of geo-plate tectonics is concerned. Judging from the rich history of the introduction of new ideas, we should say that, we do not expect a smooth passage and acceptance of the present ideas but more a fierce defence against them.

Be that it may, one thing is however certain - that is, the interpretation and subsequent implication of the recession of the of the Earth-Moon system here conducted, is nothing but a logical and straight forward interpretation despite the nature of the conclusion drawn thereof. To ourself, we merely have made the simplest and logical imaginable endeavour to interpret facts from within the accepted provinces of contemporary physics by applying the sacrosanct and embellished law of conservation of angular momentum. As to whether or not the ideas should be accepted or rejected, this we shall safely leave in the hands of our contemporaries and posterity to decide.

When one traces the history of the Earth as depicted by the present ideas, they can not help but entertain the idea that it is highly likely that all the lands of the Earth most certainly emerged from beneath the waters that once covered all of the Earth-the Earth should have constituted a perfect geoid ${ }^{5}$. If one imagines the Earth's crust as comprising a firmament ${ }^{6}$, and knowing very well that beneath and within the Earth's crust lies some complex waters systems, then, the emergent picture is that where in the beginning, the waters once where above and below the firmament, the firmament once separated the waters from the waters. With the progression of time, a portion of the firmament must have emerged above the waters (which lie above the firmament) to form the lands we inhabit today and so much cherish as a sacrosanct priceless ancestral possession and heritage.

The idea of an expanding Earth, contraction Moon, receding Moon and receding Earth-Moon system has serious implications on the formation of the Earth-Moon system if one where to wind back the hand of time for such a setting. Initially, we had included a section on this but on second thoughts we felt this would lead to a difficult reading. So, we decided to separate this into a stand-alone reading where this issue of the formation of the Earth-Moon system is tackled. This reading on the formation of the Earth-Moon system shall follow the present reading. Thereafter, we shall present the final part of our three part series of papers, where the issue of whether or not data from geophysical stations around the world supports or refutes a Hubble type flow of the World's continental plates.

\subsection{Conclusion}

Assuming the correctness of the present ideas, then, we hereby make the following conclusions:

1. Spin-orbit interaction should lead to the expansion (or contraction) of not only the Earth, but other celestial bodies in the Solar system and elsewhere in the Universe. Plate tectonics is thus not expected to be peculiar to our planet but must be abundant in the cosmos for as long as planetary recession from their parent bodies is present.

2. The global contraction of the Moon which has been confirmed by NASA, may very well be driven by gravitational decompression which comes about as a result of changes in angular momentum occurring due to the loss of orbital angular momentum of the Earth-Moon system as they drift from the Sun-this lost orbital angular momentum be transferred to the spin of the respective bodies leading to changes in their physical volume. This same phenomenon must be causing the Earth to expand, leading to global plate tectonics.

3. The lost orbital kinetic energy due to the recession of the Earth-Moon system from the Sun which according to hypothesis made herein, is converted to the kinetic energy of the spin of the Earth-Moon system, most certainly is the driver of plate tectonics, the contraction of the Moon and as-well the observed recession of Moon from the Earth.

${ }^{5}$ If the Earth where completely covered by water, such an Earth is called a geoid. In more technical terms, a geoid is the equipotential surface of the Earth's gravity field which best fits, in a least squares sense, global mean sea level.

${ }^{6} \mathrm{~A}$ firmament is the apparent surface of the imaginary sphere on which celestial bodies appear to be projected. 


\subsection{Recommendations}

If the ideas propagated herein are correct (as we believe) or are to prove to tend to that end, then, we hereby make the following recommendations:

1. To improve on the values obtained for the expansion and contraction of the Earth and Moon respectively, it is necessary to drop the assumption that $\dot{\vartheta}_{\mathrm{e}}=\dot{\vartheta}_{\mathrm{m}}=\dot{\vartheta}_{\text {orb }}=0$. We have to assume that $\dot{\vartheta}_{\mathrm{e}} \neq 0, \dot{\vartheta}_{\mathrm{m}} \neq 0$ and $\dot{\vartheta}_{\text {orb }} \neq 0$. For this, one will require a gravitational theory that can handle angular variation in the gravitational potential. Though still under construction, one such theory has been presented in [35]. We are working on this. We hope to provide our improved calculation in the near future. However, we do not expect the values to be significantly far off from what we have found out in the present endeavour.

2. Measurements hoping to detect any secular expansion of the Earth must have a threshold of sensitivity of about $+0.01 \mathrm{~mm} / \mathrm{yr}$ in order to verify or falsify the present calculation. If the present calculation is proven to be incorrect by these measurements, then, our laws of conservation of angular momentum and energy may be at fault-this is highly unlikely and we wish not to think in this direction. In worst case scenario, one will have to invoke the dark matter hypothesis in-order to save these laws from the embarrassment.

3. There is need to collect data from the different stations around the World on continental drifts and check whether or not the measured movement of plates does conform to a Hubble-type flow If these data reveal a Hubble-type flow, it would be a strong indicator that the Earth may very well be expanding.

4. It is very much likely that the recession of the Earth-Moon system is not peculiar to this celestial system alone, but is a phenomenon occurring to all the Solar planets. If this were the case, it would be interesting to calculate and most certainly speculate on the possible plate tectonic activities on these planets.

5. The present calculation hinges on the measured rate of recession of the Earth-Moon system. Thus, if accurate values are to be obtained from the calculation conducted here, there is need obtain a much more accurate value of the recession rate of the Earth-Moon system from the Sun. Krasinsky and Brumberg [5] have stated the need for more accurate measurements of the recession rate of the Earth-Moon system. This certainly needs to be done, with perhaps the present work adding one more reason for doing so.

\section{References}

[1] Mantovani, R. (1909) L’antarctide, Je minstruis. La Scieence Pour Tous, 38, 595-597.

[2] R. Mantovani (1889) Les Fractures de Lécorce Terrestre et la Théorie de Laplace. Bulletin de la Société des Sciences et Arts de l’Ile de la Réunion, 4153.

[3] W. Z.W., Xu, J.Y., Jiao, Y., Fan, S.-J., He, K. and Qin, L.-Q. (2012) Finger Nail Growth Rate and Macroelement Levels Determined by ICP-OES in Healthy Chinese College Students. Polish Journal of Environmental Studies, 21, 1067-1070.

[4] Yaemsiri, S., Hou, N., Slining, M.M. and He, K. (2010) Growth Rate of Human Fingernails and Toenails in Healthy American Young Adults. Journal of the European Academy of Dermatology and Venereology, 24, 420-423. http://dx.doi.org/10.1111/j.1468-3083.2009.03426.x

[5] Krasinsky, G.A. and Brumberg, V.A. (2004) Secular Increase of Astronomical Unit from Analysis of the Major Planets Motions, and Its Interpretation. Annalen der Physik, 90, 267-288.

[6] Standish, E.M. (2005) The Astronomical Unit Now. In: Kurtz, D.W., Ed., Transits of Venus: New Views of the Solar System and Galaxy, Proceedings IAU Colloquium, Cambridge University Press, Cambridge, 163-179.

[7] J. Romm (1994) A New Forerunner for Continental Drift. Nature, 367, 407-408. http://dx.doi.org/10.1038/367407a0

[8] Li, Z.X., Bogdanova, S.V., Collins, A.S., Davidson, A., De Waele, B., Ernst, R.E., Fitzsimons, I.C.W., Fuck, R.A., Gladkochub, D.P., Jacobs, J., Karlstrom, K.E., Lul, S., Natapov, L.M., Pease, V., Pisarevsky, S.A., Thrane, K. and Vernikovsky, V. (2008) Assembly, Configuration, and Break-Up History of Rodinia: A Synthesis. Precambrian Research, 160, 179-210. http://dx.doi.org/10.1016/j.precamres.2007.04.021

[9] Wegener, A.L. (1912) Die Entstehung der Kontinente. Geologische Rundschau, 3, 276-292. http://dx.doi.org/10.1007/BF02202896

[10] Wegener, A.L. (1912) Die Entstehung der Kontinente. Petermann's Geographische Mitteilungen, 58, 185-195, 253256,305-308.

[11] du Toit, A.L. (1937) Our Wandering Continents; An Hypothesis of Continental Drifting. Oliver \& Boyd, London.

[12] Wegener, A.L. (1915) Die Entstehung der Kontinente und Ozean. 2nd Edition, Friedrich Vieweg \& Sohn, Braun- 
schweig.

[13] Wegener, A.L. (1922) Die Entstehung der Kontinente und Ozeane. 3rd Edition, Friedrich Vieweg \& Sohn, Braunschweig.

[14] Wegener, A.L. (1929) Die Entstehung der Kontinente und Ozeane. 4th edition, Friedrich Vieweg \& Sohn, Braunschweig.

[15] Hilgenberg, O.C. (1962) Palaopollagen der erde. Neues Jahrbuch für Geologie und Paläontologie, 116, 156.

[16] Hilgenberg, O.C. (1933) Vom wachsenden erdball. Giessmann and Bartsch, Berlin.

[17] Beck, A.E. (1982) Energy changes in an Expanding Earth: In the Application of Modern Physics to the Earth and Planetary Interiors. Wiley, London, 77-83.

[18] Cook, M. A. and Eardley, A.J. (1961) Energy Requirements in Terrestrial Expansion. Journal of Geophysical Research, 66, 3907-3912. http://dx.doi.org/10.1029/JZ066i011p03907

[19] Jordan, P. (1971) The Expanding Earth: Some Consequences of Dirac’s Gravitation Hypothesis. Pergamon, Oxford, 202.

[20] Scheidegger, A.E. (1982) Principles of Geodynamics. Springer-Verlag, Heidelberg. http://dx.doi.org/10.1007/978-3-642-68457-9

[21] Carey, S.W. (1975) The Expanding Earth-An Essay Review. Earth Science Reviews, 11, 105-143. http://dx.doi.org/10.1016/0012-8252(75)90097-5

[22] Stephenson, F.R. (1997) Historical Eclipses and Earth’s Rotation. Cambridge University Press, Cambridge. http://dx.doi.org/10.1017/CBO9780511525186

[23] Stephenson, F.R. and Morrison, L.V. (1995) Long-term Fluctuations in the Earth's Rotation: 700 BC to AD 1990. Phil. Trans. R. Soc. Lond. A, 351, 165-202. http://dx.doi.org/10.1098/rsta.1995.0028

[24] Nyambuya, G. G. (2013) Why the Earth Must Expand. viXra: 1212.0107v1.

[25] Wu, X., Collilieux, X., Altamimi, Z., Vermeersen, B.L.A., Gross, R.S. and Fukumori, I. (2011) Accuracy of the International Terrestrial Reference Frame Origin and Earth Expansion. Geophysical Research Letters, 38, 5. http://dx.doi.org/10.1029/2011GL047450

[26] Williams, J.G. and Boggs, D.H. (2009) Lunar Core and Mantle. What Does LLR See. In: S. Schillak, Ed., Proceedings of the 16th International Workshop on Laser Ranging, Space Research Centre, Polish Academy of Sciences.

[27] Chapront, J., Chapront-Touze, M. and Francou. G. (2002) A New Determination of Lunar Orbital Parameters, Precession Constant and Tidal Acceleration from llr Measurements. Astronomy \& Astrophysics, 387, 700-709.

[28] Pitjeva, E.V. (2005) High-Precision Ephemerids of Planets-EPM and Determinations of Some Astronomical Constants. Solar System Research, 39, 176-186. http://dx.doi.org/10.1007/s11208-005-0033-2

[29] Zwicky, F. (1933) Die Rotverschiebung von Extragalaktischen Nebeln. Helvetica Physica Acta, 6, 110-127.

[30] Anderson, J.D., Laing, P.A., Lau, E.L., Liu, A.S., Nieto, M.M. and Turyshev, S.G. (1998) Indication, from Pioneer 10/ 11, Galileo, and Ulysses Data, of an Apparent Anomalous, Weak, Long-Range Acceleration. Physical Review Letters, 81, 2858-2861. http://dx.doi.org/10.1103/PhysRevLett.81.2858.

[31] Anderson, J.D., Laing, P.A., Lau, E.L., Liu, A.S., Nieto, M.M. and Turyshev, S.G. (2002) Study of the Anomalous Acceleration of Pioneer 10 and 11. Physical Review D, 65, arXiv: gr-qc/0104064. http://dx.doi.org/10.1103/PhysRevD.65.082004

[32] Anderson, J.D., Campbell, J.K., Ekelund, J.E., Ellis, J. and Jordan, J.F. (2008) Anomalous Orbital-Energy Changes Observed during Spacecraft Flybys of Earth. Physical Review Letters, 100, 091102. http://dx.doi.org/10.1103/PhysRevLett.100.091102

[33] Anderson, J.D. and Neito, M.M. (2009) Astrometric Solar-System Anomalies. In: Klioner, S. and Seidelmann, P.K. and Soffel, M., Eds., Relativity in Fundamental Astronomy: Dynamics, Reference Frames, and Data Analysis, Proceedings IAU Symposium, International Astronomical Union, 165.

[34] Einstein, A. (1916) Die Grundlage der Allgemeinen Relativitätstheorie. Annalen der Physik, 354, 769-822. http://dx.doi.org/10.1002/andp.19163540702

[35] Nyambuya, G.G. (2010) Azimuthally Symmetric Theory of Gravitation (I)—On the Perihelion Precession of Planetary Orbits. MNRAS, 403, 1381-1391. http://dx.doi.org/10.1111/j.1365-2966.2009.16196.x

[36] Taylor, J.R. (1982) Introduction to Error Analysis. University Science Books, Sausalito.

[37] E.M. Standish and J.C. Williams (2010) Orbital Ephemerides of the Sun, Moon, and Planets. Explanatory Supplement to the Astronomical Almanac, Chapter 8, International Astronomical Union Commission, 1381-1391.

[38] Watters, T.R., Robinson, M.S., Beyer, R.A., Banks, M.E., Bell III, J.F., Pritchard, M.E., Hiesinger, H., van der Bogert, 
C.H., Thomas, P.C., Turtle, E.P. and Williams, N.R. (2010) Evidence of Recent Thrust Faulting on the Moon Revealed by the Lunar Reconnaissance Orbiter Camera. Science, 329, 936-939. http://dx.doi.org/10.1126/science.1189590

[39] Binder, A.B. and Gunga, H.-C. (1985) Morphometric Analysis of Small-Scale Lobate Scarps on the Moon Using Data from the Lunar Reconnaissance Orbiter. Icarus, 117, 421.

[40] Banks, M.E., Watters, T.R., Robinson, M.S., Tornabene, L.L., Tran, T., Ojha, L. and Williams, N.R. (2012) Morphometric Analysis of Small-Scale Lobate Scarps on the Moon Using Data from the Lunar Reconnaissance Orbiter. Journal of Geophysical Research, 117, 11. http://dx.doi.org/10.1029/2011JE003907

[41] Watters, T.R. and Robinson, M.S. and Banks, M.E. and Tran, T. and Denevi, B.W. (2012) Recent Extensional Tectonics on the Moon Revealed by the Lunar Reconnaissance Orbiter Camera. Nature Geoscience, 329, 936-939.

[42] Turcotte, D.L. and Schubert, G. (2002) Geodynamics. Cambridge University Press, Cambridge. http://dx.doi.org/10.1017/CBO9780511807442

[43] Pollack, H.N., Hurter, S.J. and Johnson, J. R. (1993) Heat Flow from the Earth’s Interior: Analysis of the Global Data Set. Reviews of Geophysics, 31, 267-280. 


\section{Appendix A: Calculation}

We have:

$$
\begin{aligned}
L= & \mathcal{J}_{\text {orb }}\left(\sin \vartheta_{\text {orb }} \cos \vartheta_{\mathrm{m}}-\cos \vartheta_{\text {orb }} \sin \vartheta_{\mathrm{m}}\right)-\left(\mathcal{J}_{\mathrm{rm}}+\mathcal{J}_{\mathrm{e}}+\mathcal{J}_{\mathrm{m}}\right) \sin \vartheta_{\mathrm{m}} \\
& -\mathcal{S}_{\mathrm{e}}\left(\sin \vartheta_{\mathrm{e}} \cos \vartheta_{\mathrm{m}}-\cos \vartheta_{\mathrm{e}} \sin \vartheta_{\mathrm{m}}\right) .
\end{aligned}
$$

Since $\delta L=0$, it follows that:

$$
\begin{aligned}
& \mathcal{J}_{\text {orb }}\left(\frac{\delta \mathcal{J}_{\text {orb }}}{\mathcal{J}_{\text {orb }}}\right)\left(\sin \vartheta_{\text {orb }} \cos \vartheta_{\mathrm{m}}-\cos \vartheta_{\text {orb }} \sin \vartheta_{\mathrm{m}}\right)-3 \mathcal{J}_{\text {rm }}\left(\frac{\delta \mathcal{J}_{\text {rm }}}{\mathcal{J}_{\text {rm }}}\right) \sin \vartheta_{\mathrm{m}} \\
& -\mathcal{S}_{\mathrm{e}}\left(\frac{\delta \mathcal{S}_{\mathrm{e}}}{\mathcal{S}_{\mathrm{e}}}\right)\left(\sin \vartheta_{\mathrm{e}} \cos \vartheta_{\mathrm{m}}-\cos \vartheta_{\mathrm{e}} \sin \vartheta_{\mathrm{m}}\right)=0 .
\end{aligned}
$$

In the above, we have made use of the verifiable fact that $\delta \mathcal{J}_{\mathrm{rm}}+\delta \mathcal{J}_{\mathrm{e}}+\delta \mathcal{J}_{\mathrm{m}}=3 \delta \mathcal{J}_{\mathrm{rm}}$. Now, writing $\delta \mathcal{S}_{\mathrm{e}} / \mathcal{S}_{\mathrm{e}}$ as the subject of the formula, we will have:

$$
\begin{aligned}
\frac{\delta \mathcal{S}_{\mathrm{e}}}{\mathcal{S}_{\mathrm{e}}} & =\frac{\mathcal{J}_{\text {orb }}}{\mathcal{S}_{\mathrm{e}}}\left(\frac{\delta \mathcal{J}_{\text {orb }}}{\mathcal{J}_{\text {orb }}}\right) \frac{\sin \vartheta_{\text {orb }} \cos \vartheta_{\mathrm{m}}-\cos \vartheta_{\text {orb }} \sin \vartheta_{\mathrm{m}}}{\sin \vartheta_{\mathrm{e}} \cos \vartheta_{\mathrm{m}}-\cos \vartheta_{\mathrm{e}} \sin \vartheta_{\mathrm{m}}} \\
& -\frac{\mathcal{J}_{\mathrm{rm}}}{\mathcal{S}_{\mathrm{e}}}\left(\frac{\delta \mathcal{J}_{\mathrm{rm}}}{\mathcal{J}_{\mathrm{rm}}}\right) \frac{3 \sin \vartheta_{\mathrm{m}}}{\sin \vartheta_{\mathrm{e}} \cos \vartheta_{\mathrm{m}}-\cos \vartheta_{\mathrm{e}} \sin \vartheta_{\mathrm{m}}}
\end{aligned}
$$

Since $\mathcal{S}_{\mathrm{e}}=2 \pi \mathcal{M}_{\mathrm{e}} \mathcal{R}_{\mathrm{e}}^{2} / \mathcal{T}_{\mathrm{e}}, \mathcal{J}_{\text {orb }}=2 \pi\left(\mathcal{M}_{\mathrm{e}}+\mathcal{M}_{\mathrm{m}}\right) \mathcal{R}_{\text {orb }}^{2} / \mathcal{T}_{\text {orb }} \quad$ and $\quad \mathcal{S}_{\mathrm{m}}=2 \pi \mathcal{M}_{\mathrm{m}} \mathcal{R}_{\mathrm{m}}^{2} / \mathcal{T}_{\mathrm{m}}$ and assuming $\delta \mathcal{M}_{\mathrm{e}} \simeq \delta \mathcal{M}_{\mathrm{m}} \simeq 0$; using these facts to split the terms $\delta \mathcal{S}_{\mathrm{e}} / \mathcal{S}_{\mathrm{e}}, \delta \mathcal{J}_{\text {orb }} / \mathcal{J}_{\text {orb }}$ and $\delta \mathcal{S}_{\mathrm{m}} / \mathcal{S}_{\mathrm{m}}$, it follows that:

$$
\begin{aligned}
& \frac{\delta \mathcal{R}_{\mathrm{e}}}{\mathcal{R}_{\mathrm{e}}}-\frac{1}{2} \frac{\delta \mathcal{T}_{\mathrm{e}}}{\mathcal{T}_{\mathrm{e}}} \\
& =\frac{\mathcal{J}_{\text {orb }}}{\mathcal{S}_{\mathrm{e}}}\left(\frac{\delta \mathcal{R}_{\text {orb }}}{\mathcal{R}_{\mathrm{orb}}}-\frac{1}{2} \frac{\delta \mathcal{T}_{\text {orb }}}{\mathcal{T}_{\text {orb }}}\right) \frac{\sin \vartheta_{\text {orb }} \cos \vartheta_{\mathrm{m}}-\cos \vartheta_{\text {orb }} \sin \vartheta_{\mathrm{m}}}{\sin \vartheta_{\mathrm{e}} \cos \vartheta_{\mathrm{m}}-\cos \vartheta_{\mathrm{e}} \sin \vartheta_{\mathrm{m}}} \\
& -\frac{\mathcal{J}_{\mathrm{rm}}}{\mathcal{S}_{\mathrm{e}}}\left(\frac{\delta r_{\mathrm{em}}}{r_{\mathrm{em}}}-\frac{1}{2} \frac{\delta \mathcal{T}_{\mathrm{em}}}{\mathcal{T}_{\mathrm{em}}}\right) \frac{3 \sin \vartheta_{\mathrm{m}}}{\sin \vartheta_{\mathrm{e}} \cos \vartheta_{\mathrm{m}}-\cos \vartheta_{\mathrm{e}} \sin \vartheta_{\mathrm{m}}} .
\end{aligned}
$$

Now, we have to apply the Law of Conservation of Energy as laid down in (4) and (5). The Earth spins with a kinetic energy $K_{\text {spin }}^{\mathrm{e}}=2 \pi^{2} \mathcal{M}_{\mathrm{e}} \mathcal{R}_{\mathrm{e}}^{2} / \mathcal{T}_{\mathrm{e}}^{2}$. Its total stored gravitational potential energy $U_{\mathrm{g}}^{\mathrm{e}}=-G \mathcal{M}_{\mathrm{e}}^{2} / \mathcal{R}_{\mathrm{e}}^{2}$. Assuming a non-variable $G$ and mass of the Earth $\mathcal{M}_{\mathrm{e}}$, if the spin kinetic energy $K_{\text {spin }}^{\mathrm{e}}$ and the total gravitational potential energy of the Earth $U_{\mathrm{g}}^{\mathrm{e}}$ are conserved $\left(E_{\text {spin }}=K_{\text {spin }}^{\mathrm{e}}+U_{\mathrm{g}}^{\mathrm{e}}\right.$, that is $\delta E_{\text {spin }}=0$ ), then $K_{\text {spin }}^{\mathrm{e}}\left(\delta K_{\text {spin }}^{\mathrm{e}} / K_{\text {spin }}^{\mathrm{e}}\right)+U_{\mathrm{g}}^{\mathrm{e}}\left(\delta U_{\mathrm{g}}^{\mathrm{e}} / U_{\mathrm{g}}^{\mathrm{e}}\right)=0$, then from this it follows that $\delta \mathcal{T}_{\mathrm{e}} / \mathcal{T}_{\mathrm{e}}=\left(1-U_{\mathrm{g}}^{\mathrm{e}} / 2 K_{\text {spin }}^{\mathrm{e}}\right) \delta \mathcal{R}_{\mathrm{e}} / \mathcal{R}_{\mathrm{e}}$. Applying the very same assumptions to the Earth-Moon system's Solar gravitational potential energy $U_{\mathrm{g}}^{\mathrm{em}}=-G \mathcal{M}_{\odot}\left(\mathcal{M}_{\mathrm{e}}+\mathcal{M}_{\mathrm{m}}\right) / \mathcal{R}_{\text {orb }}$ the kinetic energy of its spin $K_{\text {spin }}^{\mathrm{m}}=2 \pi^{2}\left(\mathcal{M}_{\mathrm{e}}+\mathcal{M}_{\mathrm{m}}\right) \mathcal{R}_{\text {orb }}^{2} / \mathcal{T}_{\text {orb }}^{2}$, one obtains $\delta \mathcal{T}_{\mathrm{em}} / \mathcal{T}_{\mathrm{em}}=\left(1-U_{\mathrm{g}}^{\mathrm{em}} / 2 K_{\mathrm{spin}}^{\mathrm{em}}\right) \delta r_{\mathrm{em}} / r_{\mathrm{em}}$. The orbital kinetic energy $K_{\mathrm{orb}}^{\mathrm{em}}$ of the Earth-Moon system is conserved independently from the gravitational potential energy of the Earth-Moon system, that is $\delta K_{\text {orb }}^{\mathrm{em}}=0 \Rightarrow \delta \mathcal{T}_{\text {orb }} / \mathcal{T}_{\text {orb }}=\delta \mathcal{R}_{\text {orb }} / \mathcal{R}_{\text {orb }}$. Piecing everything together, we obtain:

$$
\begin{aligned}
& \frac{1}{\alpha_{\mathrm{e}}} \frac{\delta \mathcal{R}_{\mathrm{e}}}{\mathcal{R}_{\mathrm{e}}} \\
= & \frac{\mathcal{J}_{\mathrm{orb}}}{\mathcal{S}_{\mathrm{e}}}\left(\frac{\sin \vartheta_{\mathrm{orb}} \cos \vartheta_{\mathrm{m}}-\cos \vartheta_{\mathrm{orb}} \sin \vartheta_{\mathrm{m}}}{\sin \vartheta_{\mathrm{e}} \cos \vartheta_{\mathrm{m}}-\cos \vartheta_{\mathrm{e}} \sin \vartheta_{\mathrm{m}}}\right) \frac{\delta \mathcal{R}_{\mathrm{orb}}}{\mathcal{R}_{\mathrm{orb}}} \\
- & \frac{1}{\alpha_{\mathrm{em}}} \frac{\mathcal{J}_{\mathrm{rm}}}{\mathcal{S}_{\mathrm{e}}}\left(\frac{3 \sin \vartheta_{\mathrm{m}}}{\sin \vartheta_{\mathrm{e}} \cos \vartheta_{\mathrm{m}}-\cos \vartheta_{\mathrm{e}} \sin \vartheta_{\mathrm{m}}}\right) \frac{\delta r_{\mathrm{em}}}{r_{\mathrm{em}}}
\end{aligned}
$$

where $1 / \alpha_{\mathrm{e}}=1+U_{\mathrm{g}}^{\mathrm{e}} / 2 K_{\text {spin }}^{\mathrm{e}}=-(2.91 \pm 0.08) \times 10^{2}$ and $1 / \alpha_{\mathrm{em}}=1+U_{\mathrm{g}}^{\mathrm{em}} / 2 K_{\text {spin }}^{\mathrm{m}}=-(1.319 \pm 0.001) \times 10^{5}$. The above equation can be written as: 


$$
\begin{aligned}
& \frac{\delta \mathcal{R}_{\oplus}}{\mathcal{R}_{\oplus}}=\overbrace{\alpha_{\mathrm{e}}\left(\frac{\mathcal{J}_{\text {orb }}}{\mathcal{S}_{\mathrm{e}}}\right)\left(\frac{\sin \vartheta_{\text {orb }} \cos \vartheta_{\mathrm{m}}-\cos \vartheta_{\text {orb }} \sin \vartheta_{\mathrm{m}}}{\sin \vartheta_{\mathrm{e}} \cos \vartheta_{\mathrm{m}}-\cos \vartheta_{\mathrm{e}} \sin \vartheta_{\mathrm{m}}}\right) \frac{\delta \mathcal{R}_{\text {orb }}}{\mathcal{R}_{\text {orb }}}}^{\text {Solar Recessional Contribution }} \\
& -\underbrace{\frac{\alpha_{\mathrm{e}}}{\alpha_{\mathrm{em}}}\left(\frac{\mathcal{J}_{\mathrm{rm}}}{\mathcal{S}_{\mathrm{e}}}\right)\left(\frac{3 \sin \vartheta_{\mathrm{m}}}{\sin \vartheta_{\mathrm{e}} \cos \vartheta_{\mathrm{m}}-\cos \vartheta_{\mathrm{e}} \sin \vartheta_{\mathrm{m}}}\right) \frac{\delta r_{\mathrm{em}}}{r_{\mathrm{em}}}}_{\text {Lunar Recessional Contribution }},
\end{aligned}
$$

where, as usual, $\mathcal{R}_{\oplus}=\mathcal{R}_{\mathrm{e}}$ is the current radius of the Earth. If one defines $L_{\mathrm{m}}=\cos \vartheta_{\mathrm{e}} \mathcal{L}_{x}-\sin \vartheta_{\mathrm{e}} \mathcal{L}_{y}$ as has been done in (11), then, in exactly the same manner as has been conducted above, if one where to calculate the corresponding formula for the Moon system by making $\delta \mathcal{R}_{\mathrm{m}} / \mathcal{R}_{\mathrm{m}}$ of the formula, they would obtain:

$$
\begin{aligned}
& \frac{\delta \mathcal{R}_{\mathrm{m}}}{\mathcal{R}_{\mathrm{m}}}=\overbrace{-\alpha_{\mathrm{m}}\left(\frac{\mathcal{J}_{\text {orb }}}{\mathcal{S}_{\mathrm{m}}}\right)\left(\frac{\sin \vartheta_{\text {orb }} \cos \vartheta_{\mathrm{e}}-\cos \vartheta_{\text {orb }} \sin \vartheta_{\mathrm{e}}}{\sin \vartheta_{\mathrm{m}} \cos \vartheta_{\mathrm{e}}-\cos \vartheta_{\mathrm{m}} \sin \vartheta_{\mathrm{e}}}\right) \frac{\delta \mathcal{R}_{\text {orb }}}{\mathcal{R}_{\text {orb }}}}^{\text {Solar Recessional Contribution }} \\
& +\underbrace{\frac{\alpha_{\mathrm{m}}}{\alpha_{\mathrm{em}}}\left(\frac{\mathcal{J}_{\mathrm{rm}}}{\mathcal{S}_{\mathrm{m}}}\right)\left(\frac{3 \sin \vartheta_{\mathrm{e}}}{\sin \vartheta_{\mathrm{m}} \cos \vartheta_{\mathrm{e}}-\cos \vartheta_{\mathrm{m}} \sin \vartheta_{\mathrm{e}}}\right) \frac{\delta r_{\mathrm{em}}}{r_{\mathrm{em}}}}_{\text {Lunar Recessional Contribution }} .
\end{aligned}
$$

This completes our derivation. 